\title{
An irregular narrow complex tachycardia
}

\author{
Xiaodong Zhang ${ }^{1}$, Ruike Yang ${ }^{1}$, and Luigi Di Biase ${ }^{1}$ \\ ${ }^{1}$ Montefiore Medical Center
}

October 30, 2020

\section{Xiaodong Zhang ${ }^{1}$, MD, PhD, Ruike Yang ${ }^{12}$, MD, Luigi Di Biase ${ }^{1}$, MD, PhD, FHRS}

1 Division of Cardiology, Department of Medicine, Montefiore Medical Center, Albert Einstein College of Medicine, Bronx, New York

2 Division of Cardiology, Department of Medicine, Henan Provincial People's Hospital, Zhengzhou, China

Correspondence : Dr Luigi Di Biase, Montefiore Medical Center, Albert Einstein College of Medicine, 111 East 210th St, Bronx, NY 10467. E-mail address: dibbia@gmail.com .

Keywords : narrow complex tachycardia, dual atrioventricular nodal non-re-entrant tachycardia, dual atrioventricular nodal re-entrant tachycardia, autonomic nervous system

\section{Disclosure statement}

The authors declare that there are no conflicts of interest.

\section{Case presentation}

A 53 year-old gentleman with hypertension presented to the emergence room with sudden chest pain, associated with shortness of breath and dizziness. His 12-lead electrocardiograms (ECG) showed an irregular narrow complex tachycardia with frequent wide QRS complexes. (Figure 1). Echocardiogram revealed normal left ventricular function without structural abnormalities.

\section{Discussion}

His 12-lead electrocardiograms (ECG) exhibited an irregular rhythm characterized by the following features: 1) dual atrioventricular (AV) node physiology, two families of PR intervals, 2) discernible p waves with morphology consistent with sinus rhythm, 3) some of the p waves followed by two QRS complexes, 4) multiple wide QRS complexes manifesting variable right bundle branch block (RBBB) aberrancy (Figure 1).

During electrophysiology study (EPS), baseline AV nodal retrograde conduction was present, concentric and decremental in nature. Dual AV nodal physiology was demonstrated by a 69 ms atrio-his (AH) "jump" during atrial stimulation with single extra beat. No arrhythmia could be induced, so isoproterenol $(10 \mathrm{mcg} / \mathrm{min})$ was administrated. Spontaneously, a premature p wave traverses down both fast (FP) and slow pathways (SP) simultaneously causing 1 to 2 initiation of a narrow complex tachycardia (Figure 2), which was confirmed as typical slow-fast atrioventricular nodal re-entrant tachycardia (AVNRT) by RV overdriving pacing: (1) VAV response, (2) post pacing interval - tachycardia cycle length $=276 \mathrm{~ms}$. Later, an irregular narrow complex tachycardia was observed, every p wave provoked two ventricular excitations with preceding His deflections. Moreover, the rhythm pattern was not fixed, from constant 1:2 AV conduction to intermittent SP block or both pathways block (Figure 3). After radiofrequency ablation in the SP region, the incessant tachycardia was abolished. 
Dual atrioventricular nodal non-re-entrant tachycardia (DAVNNT) caused by simultaneous dual antegrade nodal conduction (also termed "double-fire") is an uncommon form of dual AV nodal tachycardia. It is even more unique to find a patient presents with both AVNRT and DAVNNT, which was considered theoretically impossible. It was believed that there are two prerequisites for the occurrence of DAVNNT: (1) the difference in conduction times over the FP and SP should be long enough to exceed the refractory period of the lower common pathway and/or His-Purkinje fibers. (2) retrograde conduction should be absent or poor in both pathways, allowing sustained anterograde conductions along both pathways $[1,2]$. The hypothesis makes AVNRT unlikely as retrograde conduction is necessary for re-entrant circuit. There have only been few reports of a co-existence of AVNRT and dual antegrade conduction [3,4]. In this case, retrograde FP conduction presents at baseline, but AVNRT cannot be induced until sympathetic stimulation changed the balance between retrograde fast and anterograde slow pathway conduction.

Also in our case, dual antegrade conduction exhibited a wide spectrum of presentations. In figure $3 \mathrm{~A}, \mathrm{FP}$ conduction (A1H1) prolonged the SP conduction (A1H2) by its retrograde concealed penetration of the SP, making it long enough to exceed the refractory period of His-Purkinje system. Possible Mobitz type 1 AV block (Wenckebach) conduction along FP (A2H3), which may just reflect the decremental properties of the distal common pathway. However, the following FP (A3H4) conduction time recovered instead of block, which can better be explained by retrograde concealed conduction from the opposite pathway. Because A2 didn't conduct down the SP, there was no retrograde concealed conduction to FP, explaining why the short A3H4 interval. Similarly, in figure 3B, the SP impulse (A1H2) had concealed retrograde conduction into the next FP conduction (A2H3), and likewise A2H3 prolonged the next SP conduction (A2H4). The influence of slowing conduction through both pathways resulted in further delayed retrograde conduction into the opposing pathway, causing the next p wave (A3) reached the refractory period of both pathways. One may argue the A3 could be just an echo beat. However, the fact that atrial activation of high right atrium (HRA) is earlier than coronary sinus (CS) and AA intervals remains the same makes it less likely. During isoproterenol washout, the balance between the two pathways changed again. We observed a constant changing rhythm mixed with DAVNNT and SP or FP only conduction. One of the prerequisite of maintaining DAVNNT should be the functional balance of bidirectional conduction between FP and SP, rather than retrograde conduction block. Autonomic nervous system plays a major role in modifying the balance of nodal conduction system, resulting in different conduction properties at different times, which makes this case possible.

In our case there are variable aberrant QRS morphologies, which can be understood based on the $\mathrm{HH}$ intervals preceding the narrow versus the aberrant complexes. RBBB aberration is functional, as a result of a long preceding $\mathrm{HH}$ interval following by a short cycle ("rate-dependent aberrancy") (Figure 3A).

It is tricky to differentiate DAVNNT from junctional extrasystoles. But the possibility of a junctional extrasystole with retrograde block is very unlikely given the association between atrial excitations and His depolarizations. Besides, abolition of arrhythmia by SP ablation seems to be another strong proof of the diagnosis. Both AVNRT and DAVNNT respond to SP region ablation.

\section{Figure legends}

Figure 1. Both A and B are presenting 12-lead electrocardiography (ECG).

Figure 2. A premature p wave traverses down both fast pathway and slow pathway simultaneously causing 1 to 2 initiation of a narrow complex tachycardia. The green arrows indicate the fast pathway conduction, and the red arrows indicate the slow pathway conduction.

Figure 3. Intracardiac tracings of the double-fire phenomenon. The green arrows indicate the fast pathway conduction, and the red arrows indicate the slow pathway conduction. A A1H1=82 ms, A1H2=339 ms, $\mathrm{A} 2 \mathrm{H} 3=150 \mathrm{~ms}, \mathrm{~A} 3 \mathrm{H} 4=88 \mathrm{~ms}, \mathrm{~A} 3 \mathrm{H} 5=400 \mathrm{~ms}, \mathrm{H} 0 \mathrm{H} 1=504 \mathrm{~ms}, \mathrm{H} 1 \mathrm{H} 2=255 \mathrm{~ms}, \mathrm{H} 2 \mathrm{H} 3=328 \mathrm{~ms}$, AA intervals $=500 \mathrm{~ms} . \mathbf{B} \mathrm{A} 1 \mathrm{H} 1=73 \mathrm{~ms}, \mathrm{~A} 1 \mathrm{H} 2=356 \mathrm{~ms}, \mathrm{~A} 2 \mathrm{H} 3=181 \mathrm{~ms}, \mathrm{~A} 2 \mathrm{H} 4=438 \mathrm{~ms}$, AA intervals $=502 \mathrm{~ms}$.

\section{References}


1 Kim SS, Lai R, Ruffy R. Paroxysmal nonreentrant supraventricular tachycardia due to simultaneous fast and slow pathway conduction in dual atrioventricular node pathways. J Am Coll Cardiol 1987;10:456-461.

2 Wang N. Dual atrioventricular nodal nonreentrant tachycardia: a systematic review. Pacing Clin Electrophysiol 2011;34:1671-81.

3 Anselme F, Frederiks J, Boyle NG, Papageorgiou P, Josephson ME. An unusual cause of tachycardiainduced myopathy. Pacing Clin Electrophysiol 1996;19:115-911.

4 Hartmann J, Jungen C, Stec S, Klatt N, Willems S, Makimoto H, Steven D, Purerfellner H, Martinek M, Meyer C. Outcomes in patients with dual antegrade conduction in the atrioventricular node: insights from a multicentre observational study. Clin Res Cardiol 2020;109(8):1025-1034

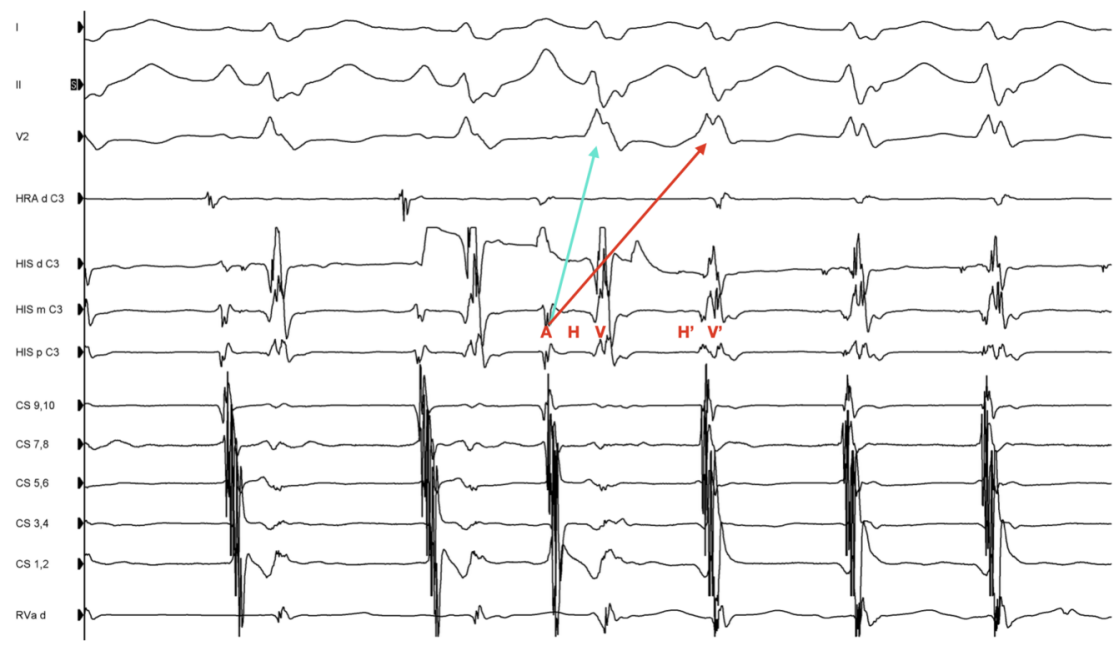




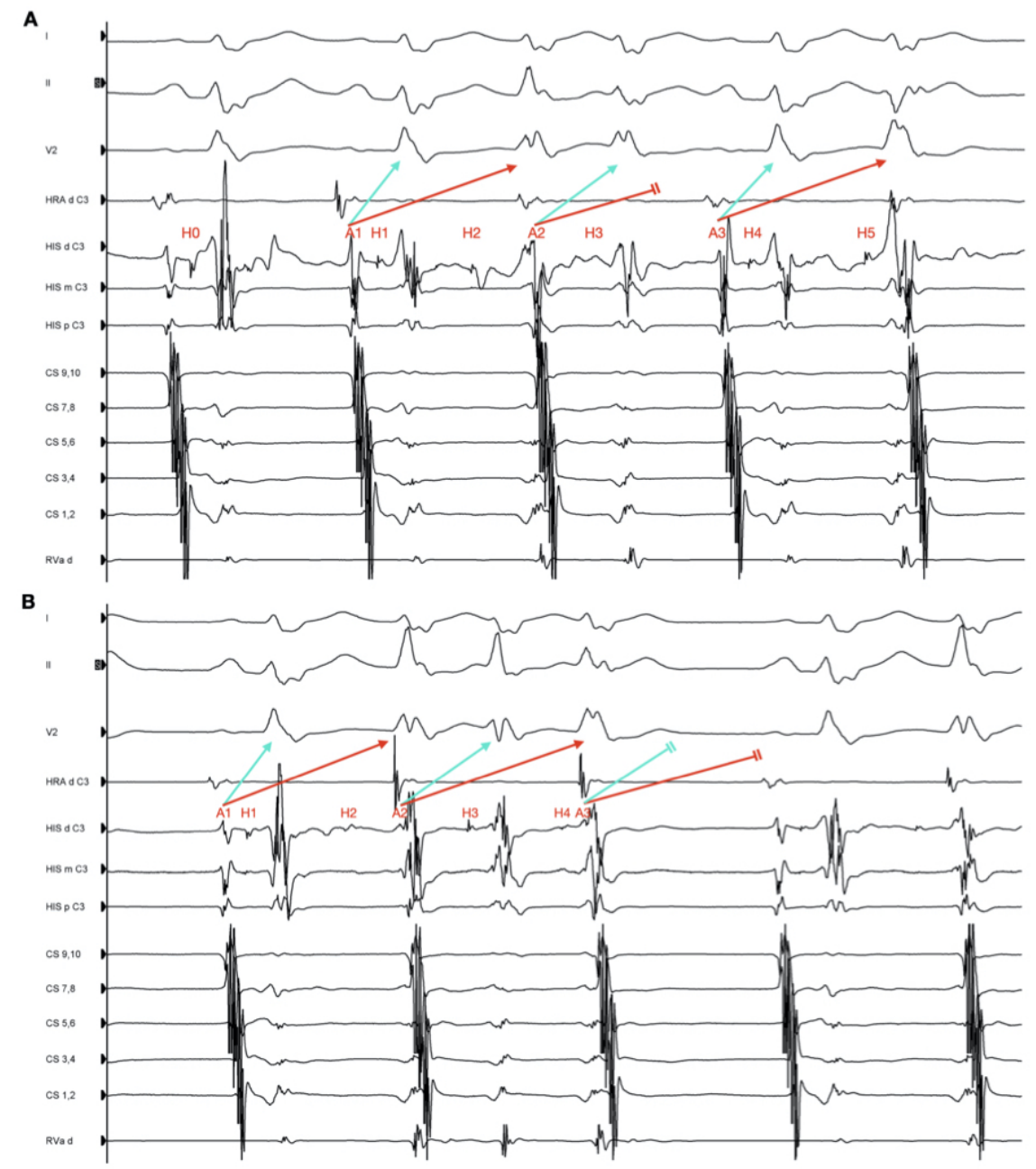


A

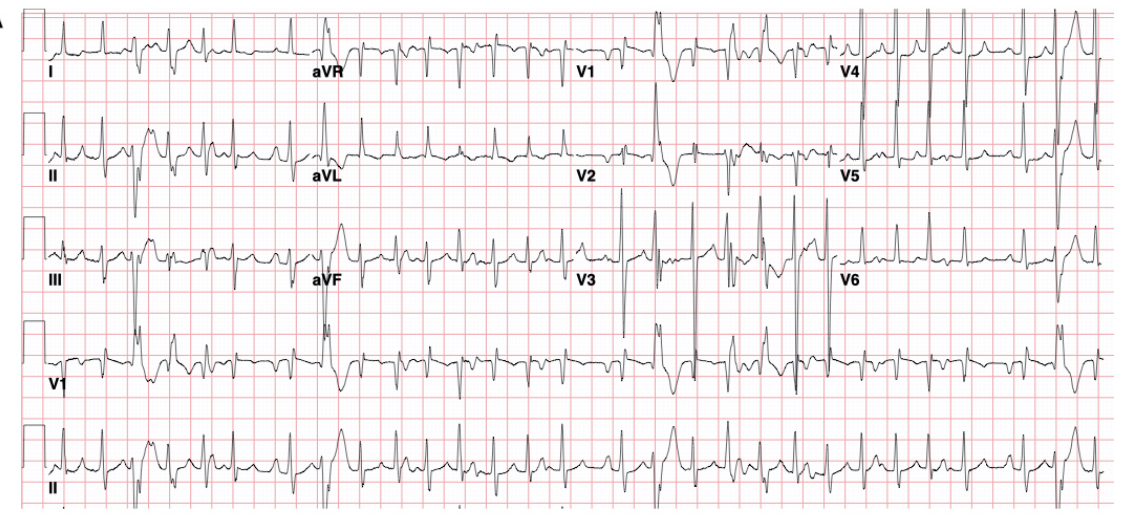

B

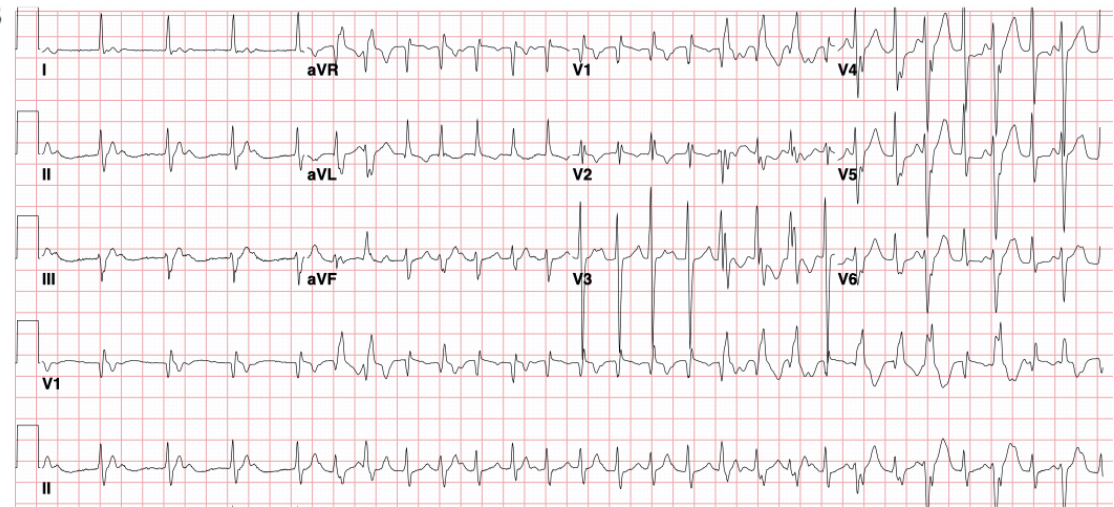

